\title{
Are the Wear and Osteolysis Outcomes Different between Annealed and Remelted First-Generation Highly Crosslinked Polyethylene after Long-Term Implantation?
}

\author{
Jaclyn T. Schachtner ${ }^{1}$ Daniel W. MacDonald, MS ${ }^{20}$ Gregg R. Klein ${ }^{3}$ Arthur L. Malkani ${ }^{4}$ \\ Matthew Kraay ${ }^{5}$ Clare M. Rimnac $^{6}$ Michael A. Mont ${ }^{7}$ Gwo-Chin Lee ${ }^{8}$ Steven Michael Kurtz ${ }^{1,2}$
}

${ }^{1}$ Department of Biomedical Engineering, Exponent Inc., Philadelphia, Pennsylvania

2 Implant Research Core, Drexel University School of Biomedical Engineering Science and Health Systems, Philadelphia, Pennsylvania

${ }^{3}$ Department of Orthopaedic Surgery, Rothman Institute, Montvale,

Address for correspondence Daniel W. MacDonald, MS, Implant Research Core, Drexel University School of Biomedical Engineering Science and Health Systems, 3401 Market St., Suite 345, Philadelphia, New Jersey

${ }^{4}$ Department of Orthopedics, University of Louisville, Louisville, Kentucky PA 19104 (e-mail: dm68@drexel.edu).

${ }^{5}$ Department of Orthopaedics, University Hospitals Cleveland

Medical Center, Cleveland, Ohio

${ }^{6}$ Center for the Evaluation of Implant Performance, Case Western Reserve University Case School of Engineering, Cleveland, Ohio

${ }^{7}$ Department of Orthopaedic Surgery, Lenox Hill Hospital at

Northwell Health, New York City, New York

8 Department of Orthopaedic Surgery, Penn Presbyterian Medical

Center, Philadelphia, Pennsylvania

J Hip Surg 2020;4:158-165.

\author{
Abstract \\ Keywords \\ - first-generation \\ HXLPE \\ - osteolysis \\ - wear \\ - total hip arthroplasty \\ - oxidation
}

First-generation highly crosslinked polyethylene (HXLPE) was developed to reduce polyethylene wear debris and subsequent osteolysis. Two thermal stabilization strategies were developed, annealing and remelting, to remove free radicals remaining in the polymer. Both types of HXLPEs have demonstrated better wear resistance to conventional polyethylene in hip arthroplasty. However, few studies have directly compared the mid- to long-term clinical outcomes of first-generation HXLPEs. We sought to address the following research questions: (1) is there a difference between the revision reasons for HXLPE formulations (annealed and remelted), (2) is there a difference in oxidation between annealed and remelted HXLPEs, (3) is there a difference in the linear penetration rate of annealed and remelted HXLPEs, and (4) does the formulation of first-generation HXLPEs affect the prevalence of osteolysis? A total of 129 firstgeneration HXLPE acetabular liners were collected in a multicenter retrieval program. These components were implanted for 5 or more years and were fabricated from annealed or remelted HXLPE. Reasons for revision, body mass index, age, sex, and activity levels were collected from medical records. Oxidation was measured at four regions of interest: bearing surface, backside surface, locking mechanism, and rim. Liner penetration was directly measured from retrievals using a micrometer. Osteolysis was reported in the operative notes by the revising surgeon and a thorough review of the operative notes and radiographs. Revision reasons included infection, instability, pain, and loosening. The annealed liners had higher oxidation indices than remelted received

May 15, 2020

accepted

July 21,2020

published online

September 17, 2020
Copyright $\odot 2020$ by Thieme Medical

Publishers, Inc., 333 Seventh Avenue, New York, NY 10001, USA Tel: +1(212) 760-0888
DOI https://doi.org/ 10.1055/s-0040-1716576. ISSN 2472-8446. 
liners. There was no difference in linear penetration rates between cohorts. There was no difference in osteolysis prevalence between cohorts. We found remelted HXLPE to be more oxidatively stable than annealed HXLPE but did not find a significant difference in the linear penetration rates or the prevalence of osteolysis. Our findings demonstrate sustained long-term wear resistance of both cohorts of HXLPE. We did not find evidence to support a long-term clinical difference between the formulations of HXLPE.

Highly crosslinked polyethylene (HXLPE) was clinically introduced in 1998 as a method to decrease implant wear and subsequent osteolysis. ${ }^{1}$ Crosslinking is achieved by exposure to high energy radiation. However, radiation exposure also causes the formation of free radicals within the polymer. ${ }^{1}$ Residual free radicals can lead to accelerated degradation in vivo. ${ }^{2}$ Two potential solutions were introduced for the reduction of free radicals in first-generation HXLPEs. One method was known as annealing, in which the polymer was heated to a temperature just below the melting point. ${ }^{1}$ Compared with conventional, gamma inert sterilized ultrahigh-molecularweight polyethylene (hereafter referred to as conventional UHMWPE), annealed HXLPE retains its crystallinity and some mechanical properties. Specifically, annealed HXLPE has similar yield strength and ultimate tensile strength compared with conventional UHMWPE. However, the elongation, toughness, and fatigue crack propagation resistance of annealed HXLPE are reduced when compared with conventional UHMWPE due to the higher levels of crosslinking. This method maintains measurable levels of free radicals, ${ }^{3,4}$ which can lead to oxidation of the material. The alternative method, known as remelting, heats the polymer to a temperature above its melting point, resulting in an HXLPE with an undetectable amount of residual free radicals. ${ }^{1}$ This method, however, decreases the polyethylene's static and cyclic mechanical properties compared with conventional UHMWPE. ${ }^{5}$ The crosslinking reduces the chain mobility of the polymer, which reduces the elongation and toughness and further reduces the fatigue crack propagation resistance compared with annealed HXLPE. Additionally, the remelting and subsequent recrystallization of the polymer results in a lower crystallinity (as compared with conventional UHMWPE), which confers a lower strength of the polymer. Regardless of the method used to reduce the free radicals in first-generation HXLPEs, the resulting material exhibits superior wear resistance at the expense of some mechanical properties when compared with conventional UHMWPE.

Through improved wear resistance and thus decreased generation of polyethylene debris, crosslinking of polyethylene was hypothesized to improve implant survivorship and decrease the prevalence of osteolysis. ${ }^{6}$ Kurtz et $\mathrm{al}^{3}$ reported an $87 \%$ decrease in the incidence of osteolysis of HXLPE when compared with conventional UHMWPE. Similarly, Capello et al ${ }^{6}$ reported a reduction in wear rate by $78 \%$ in annealed HXLPE when compared with conventional polyethylene. However, this study was restricted by a smaller patient cohort (38 patients) and follow-up of only 10 years. ${ }^{6}$ Several studies have attempted to capture the long-term results by following patients at a minimum of 7 to 10 years and have confirmed lower amounts of wear for remelted and annealed HXLPEs compared with conventional polyethylene. ${ }^{6-8}$ However, these studies have not evaluated the reasons for revision and whether this trend continues beyond 10 years in vivo.

To determine the clinical performance of long-term firstgeneration HXLPEs, we examined oxidation and wear rates in retrieved annealed and remelted acetabular liners that were implanted for 5 or more years. We sought to address the following research questions: (1) is there a difference between the revision reasons for HXLPE formulations (annealed and remelted), (2) is there a difference in oxidation between annealed and remelted HXLPEs, (3) is there a difference in the linear penetration rate of annealed and remelted HXLPEs, and (4) does the formulation of first-generation HXLPEs affect the prevalence of osteolysis?

\section{Materials and Methods}

\section{Patient Demographics and Implant Characterization}

Between January 2000 and July 2018, acetabular liners were collected from 14 surgical centers as a part of an institutional review board approved multi-institutional retrieval analysis program during routine revision surgery. The respective boards approved the study protocols at all participating centers. All explanted acetabular liners were cleaned using institutional procedures and expeditiously stored in a subzero freezer to minimize ex vivo oxidative changes. ${ }^{9}$

During the study period, our retrieval program received approximately 1,100 HXLPE acetabular liners, of which 790 acetabular liners were confirmed as being fabricated from a first-generation HXLPE. The scope of the study was limited to the two manufacturers from which we collected the greatest number of first-generation HXLPE acetabular liners: annealed (Crossfire, Stryker Orthopaedics); and remelted (Longevity, Zimmer Biomet). Of these 645 acetabular liners, 129 were implanted for 5 years or more. This resulted in 55 annealed acetabular liners and 74 remelted acetabular liners for inclusion in this study (-Fig. 1).

The annealed liner cohort had smaller inner diameters (median inner diameter $=28 \mathrm{~mm}$, range: $22-36 \mathrm{~mm}$ ) than the remelted cohort (median inner diameter $=32 \mathrm{~mm}$; range: $28-40 \mathrm{~mm}$; median difference $=4 \mathrm{~mm} ; p<0.001$ ). Liners from the annealed cohort had inner diameters of $22 \mathrm{~mm}(n=1), 28 \mathrm{~mm}(n=40), 32 \mathrm{~mm}(n=11)$, or $36 \mathrm{~mm}$ $(n=3)$. The remelted cohort had inner diameters of $28 \mathrm{~mm}$ 


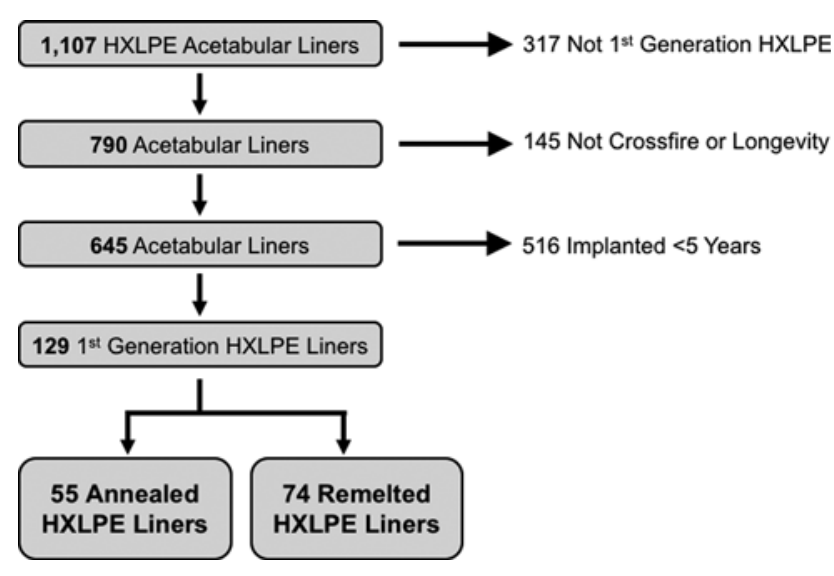

Fig. 1 Selection process of the study samples. HXLPE, highly crosslinked polyethylene.

( $n=16), 32 \mathrm{~mm}(n=37), 36 \mathrm{~mm}(n=19)$, or $40 \mathrm{~mm}(n=2)$. In the annealed cohort, the material of the femoral head was cobalt-chrome alloy in $64 \%(n=35 / 55)$ of the implants and ceramic in $36 \%(n=20 / 55)$ of the implants. In the remelted cohort, the material of the femoral head was cobalt-chrome alloy in $92 \%$ ( $n=68 / 74)$ of the implants and ceramic in $4 \%$ ( $n=3 / 74)$ of the implants. Femoral head material was not reported in three revisions in the remelted cohort.

Clinical data and operative reports were collected and used to determine patient age, sex, body mass index (BMI), implantation time, and University of California Los Angeles (UCLA) activity score ( $\mathbf{- T a b l e ~} \mathbf{1}$ ). Patient UCLA activity scores were reported for $86 \%(n=47 / 55)$ of the annealed cohort and $80 \%(n=59 / 74)$ of the remelted cohort. UCLA activity assessment was conducted through a patient questionnaire, which inquired about their activity level 3 months prior to surgery as well as their maximum activity level since implantation (on a scale of 1 to 10). The patient characteristics in the two cohorts were similar when evaluating patient age at device insertion $(p=0.94)$, sex $(p=0.84)$, BMI $(p=0.58)$, and UCLA activity score $(p=0.59)$. The annealed cohort had a longer implantation time (median $=9.8$ years; range: $5.0-17.0$ years) when compared with the remelted cohort (median $=7.8$ years; range: $5.0-16.5$ years), likely due to its earlier clinical introduction (median difference: 2.0 years; $p=0.007)$. Also, $22 \%(n=12 / 55)$ of the revised components from the annealed cohort and 23\% $(n=17 / 74)$ from the remelted cohort were retrieved from patients who had a history of at least one previous revision surgery. Reasons for revision were reported in the clinical report and also assessed based on medical records, radiographs, and examination of the retrieved components.

\section{Analyses of Oxidation}

To examine the oxidation properties, thin $(\sim 200 \mu \mathrm{m})$ slices were taken from the superior/inferior axis of the revised acetabular liners. The HXLPE slices were boiled in heptane for 6 hours to extract lipids absorbed in vivo. ${ }^{10}$ Using transmission Fourier transform infrared (FTIR) spectroscopy, 3-mm line profiles were taken perpendicular to regions of interest (ROIs), according to ASTM 2102-13. ROIs for the revised acetabular liners were the rim, locking mechanism, backside, and bearing surface. An oxidation index (OI) was calculated in accordance with ASTM 2102. ${ }^{11}$

\section{Analyses of Linear Penetration}

Wear was assessed through linear femoral head penetration using calibrated digital micrometers (accuracy of $0.001 \mathrm{~mm}$ ), where the thickness of the acetabular liners were measured in the loaded and unloaded regions. The femoral head penetration rate was determined by dividing the head penetration depth by the implantation time to calculate a penetration rate $(\mathrm{mm} / \text { year })^{12}$ for each measured liner. Seventeen (31\%) of 55 of the annealed cohort and 15 (20\%) of 74 of the remelted cohort for a total of 31 acetabular liners were excluded from this measurement due to physical damage occurring during explanation, which would cause inaccurate penetration rate measurements.

\section{Incidences of Osteolysis}

Osteolysis was determined by the revising surgeon and a detailed review of the operative notes and radiographs. Patients were identified as having osteolysis if the osteolysis was indicated as a revision reason by the revising surgeon. Additionally, we reviewed the intraoperative notes of each case and identified patients with osteolysis if the revising surgeon mentioned osteolysis or bone loss in the report.

\section{Statistical Analyses}

Using the Shapiro-Wilk test, continuous variables were tested for normality and were found to be nonnormal. Given this result, the two cohorts were tested for differences using the Wilcoxon test. Spearman's rank correlation test was used to analyze all correlations. These statistical tests were performed with an $\alpha$ value of 0.05 . All statistical tests were conducted using commercial statistical software (SPSS v. 25, IBM Corp.).

Table 1 Summary of patient demographics in annealed and remelted cohorts in intermediate and long-term implantation

\begin{tabular}{|c|c|c|c|c|c|c|c|}
\hline & Cohort & $n$ & $\begin{array}{l}\text { Age } \\
\text { (years) }\end{array}$ & $\begin{array}{l}\text { Gender } \\
(\% \mathrm{~F})\end{array}$ & $\begin{array}{l}\text { BMI } \\
\left(\mathrm{kg} / \mathrm{m}^{2}\right)\end{array}$ & $\begin{array}{l}\text { Implantation } \\
\text { time (years) }\end{array}$ & Max UCLA score \\
\hline \multirow[t]{2}{*}{ Annealed } & Intermediate term (5-10 y) & 29 & $58 \pm 15$ & $62 \%$ & $31.4 \pm 6.6$ & $7.3 \pm 1.7$ & $6(1-8)$ \\
\hline & Long term $(10+y)$ & 26 & $54 \pm 17$ & $39 \%$ & $29.1 \pm 5.9$ & $12.2 \pm 1.8$ & $7(3-10)$ \\
\hline \multirow[t]{2}{*}{ Remelted } & Intermediate term (5-10 y) & 59 & $57 \pm 16$ & $51 \%$ & $29.8 \pm 7.1$ & $7.2 \pm 1.2$ & $6(2-10)$ \\
\hline & Long term $(10+y)$ & 15 & $53 \pm 13$ & $60 \%$ & $28.2 \pm 5.2$ & $12.0 \pm 1.8$ & $7(4-10)$ \\
\hline
\end{tabular}

Abbreviations: BMI, body mass index; F, female; UCLA, University of California Los Angeles. 


\section{Results}

The primary revision reasons were different between the two cohorts ( $p=0.04$; Pearson's chi-square test). Specifically, infection was more prevalent in the remelted cohort (24\%) compared with the annealed cohort $(9 \%)(p=0.025)$. The predominant revision reasons in the annealed cohort were infection ( $9 \% ; n=5 / 55)$, instability ( $29 \% ; n=16 / 55)$, loosening $(29 \% ; n=16 / 55)$, periprosthetic fracture $(7 \% ; 4 / 55)$, and device fracture (fractured ceramic head [ $11 \% ; n=6 / 55]$ ). The remelted cohort was also principally revised for infection (24\%; $n=18 / 74)$, instability (39\%; $n=29 / 74)$, loosening (23\%; $n=17 / 74)$, periprosthetic fracture $(3 \% ; n=2 / 74)$, and device fracture (ceramic head [1\%; $n=1 / 72]$, femoral stem $[1 \% ; n=1 / 72]$ ) ( - Fig. 2 ). For the 33 reports of loosening in the two cohorts, 18 were for loosening of the acetabular shell, 11 were loosening of the femoral stem, and 4 were for both acetabular shell and femoral stem loosening.

After review of operative notes and clinical radiographs, $16 / 55$ (29\%) of the annealed cohort and $18 / 74$ (24\%) of the remelted cohort had evidence of osteolysis. However, 7 of the 16 patients with annealed HXLPE liners with osteolysis had a history of at least one prior revision. Similarly, in the remelted cohort, 9 of the 18 patients with osteolysis had a history of at least one prior revision. Therefore, in primary cases, osteolysis was similar among the patients with annealed liners (9/39 [23\%]) and remelted liners (9/56 [16\%]; $p=0.78)$. Osteolysis was listed as a revision reason in three cases in the annealed cohort and in one case in the remelted cohort.

OIs measured in the annealed cohort were higher than those measured in the remelted cohort at the bearing surface (annealed cohort median $=0.4$, interquartile range $[\mathrm{IQR}]=0.1$; remelted cohort median $=0.1, \mathrm{IQR}=0.1$; median difference $=0.3 ; p<0.001$ ), backside (annealed cohort median $=0.3$, $\mathrm{IQR}=0.2$; remelted cohort median $=0.1, \mathrm{IQR}=0.1$; median difference $=0.2 ; p<0.001$ ), locking mechanism (annealed cohort median $=0.3, \mathrm{IQR}=0.2$; remelted cohort median $=0.1$,
$\mathrm{IQR}=0.1 ;$ median difference $=0.2 ; p<0.001$ ), and rim (annealed cohort median $=5.2, \mathrm{IQR}=3.7$; remelted cohort median $=0.1, \mathrm{IQR}=0.1$; median difference $=5.1 ; p<0.001$ ) (-Fig. 3). Regional differences were observed within each of the cohorts. In the remelted cohort, the bearing surface had the largest OI compared with the backside, locking mechanism, and the rim (median differences $=0.08,0.08$, and 0.08 , respectively, and $p \leq 0.001,<0.001$, and $<0.001$, respectively). The remelted acetabular liners had similar levels of oxidation at the rim, backside, and locking mechanism $(p=1.0)$. In the annealed cohort, the rim had the greatest amount of oxidation as compared with the bearing, backside, and locking mechanism regions (median difference $=4.8,4.9$, and 4.9 , respectively, and $p \leq 0.001,<0.001$, and $<0.001$, respectively). To a lesser degree, the bearing surface had higher oxidation than the locking mechanism (median difference $=0.1 ; p=0.023$ ). In both the annealed and remelted cohorts, oxidation was not correlated with implantation time at the bearing surface (Spearman's $\rho=0.19$ and 0.03 , respectively, and $p=0.18$ and 0.78 , respectively), backside surface (Spearman's $\rho=0.13$ and -0.04 , respectively, and $p=0.35$ and 0.73 , respectively), locking mechanism (Spearman's $\rho=0.15$ and -0.03 , respectively, and $p=0.30$ and 0.79 , respectively), or the rim (Spearman's $\rho=-0.02$ and 0.00 , respectively, and $p=0.86$ and 0.99 , respectively).

Femoral head penetration rates of the annealed and remelted HXLPE acetabular liners were similar $(p=0.06$; -Fig. 4). The annealed acetabular liners had a median penetration rate of $0.014 \mathrm{~mm} /$ year (range: $0.005-0.15 \mathrm{~mm} / \mathrm{year}$; IQR: $0.02 \mathrm{~mm} /$ year). The remelted acetabular liners had a median penetration rate of $0.009 \mathrm{~mm} / \mathrm{year}$ (range: 0.000 $0.081 \mathrm{~mm} /$ year; IQR: $0.016 \mathrm{~mm} /$ year). For both the annealed and remelted cohorts, the penetration rates of liners where the patient had osteolysis were similar to liners with patients who did not have osteolysis ( $p=0.72$ and 0.10 , respectively). In both cohorts, the femoral head penetration rates were not

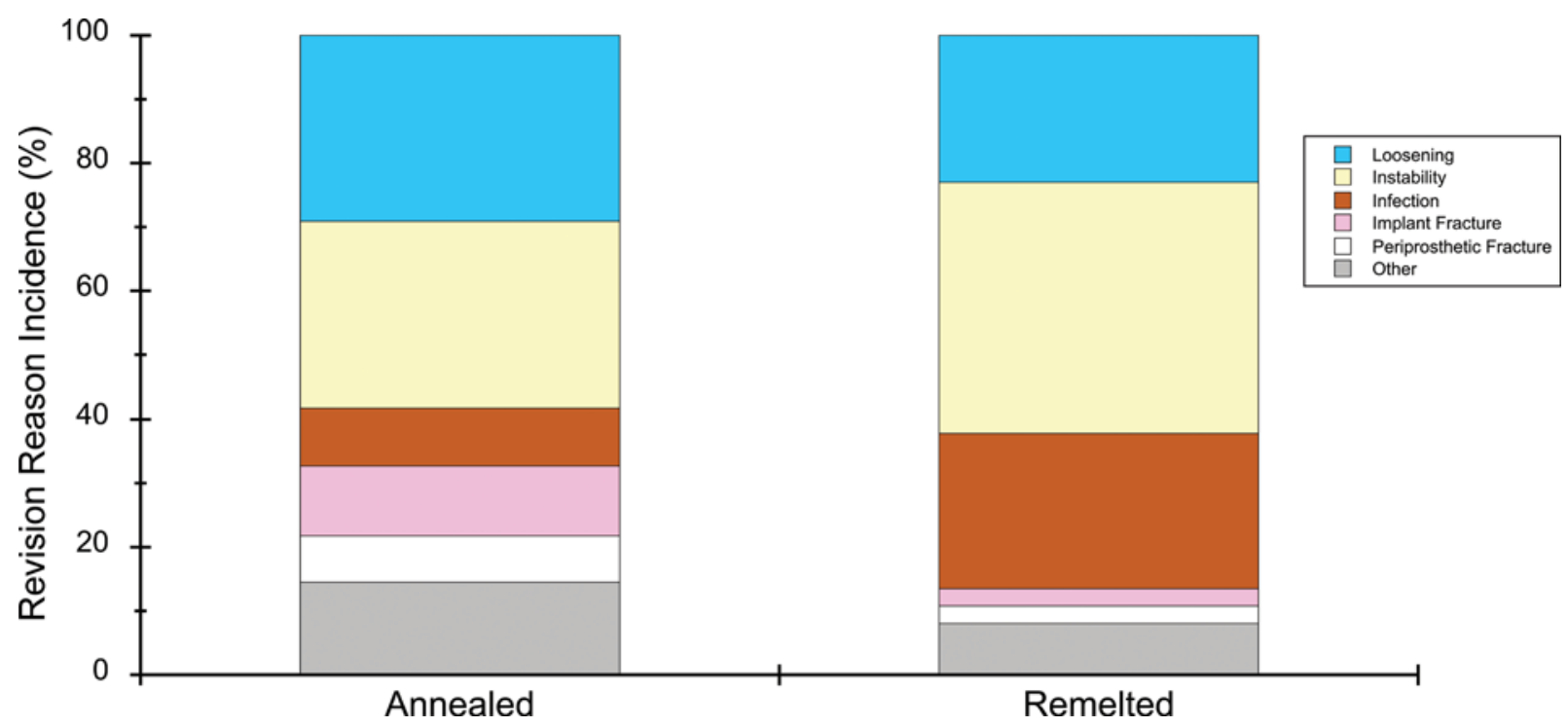

Fig. 2 Revision reasons for the annealed and remelted highly crosslinked polyethylene cohorts. 


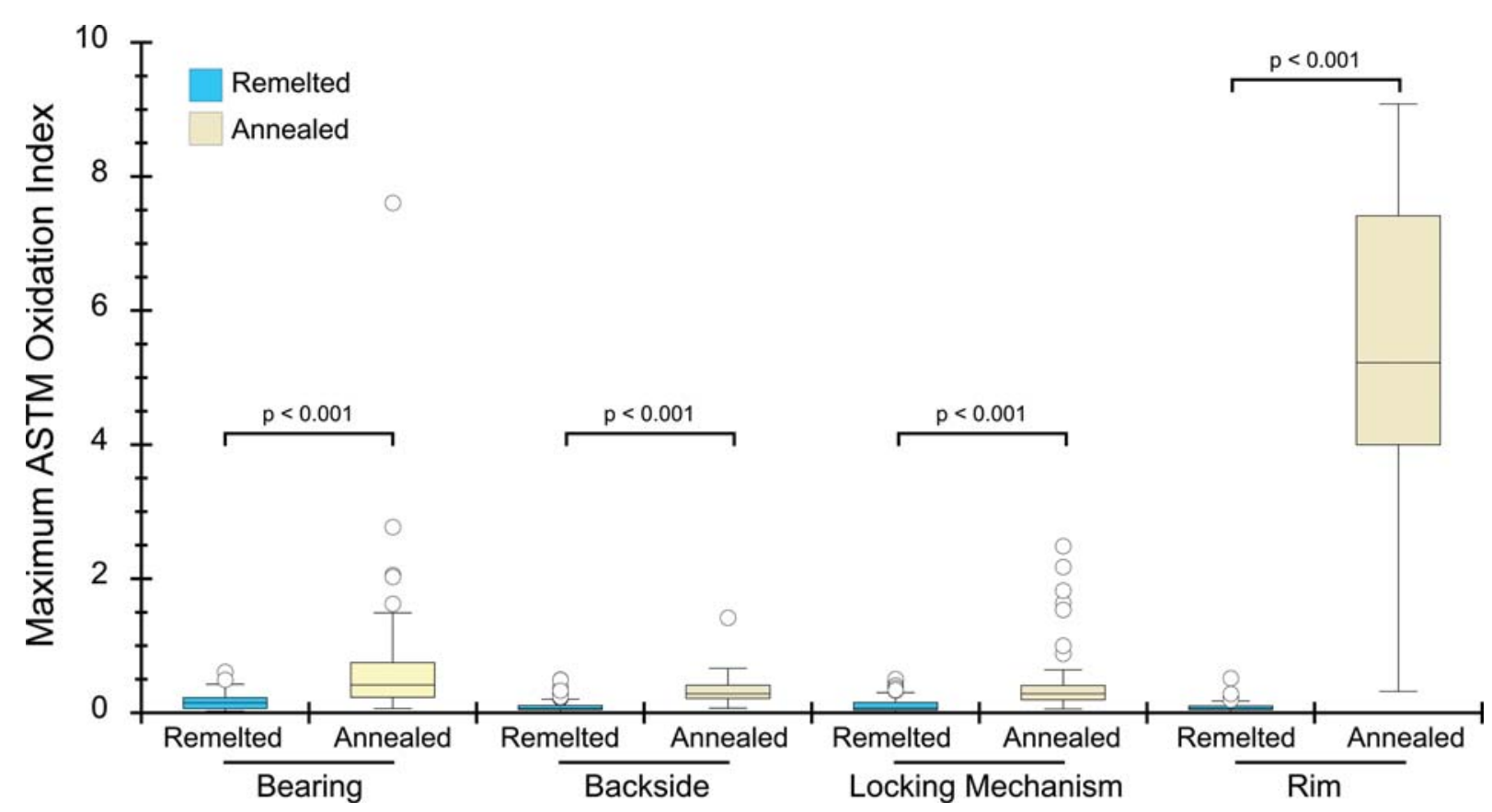

Fig. 3 Oxidation indices were higher in the annealed highly crosslinked polyethylene liners at all regions of interest.
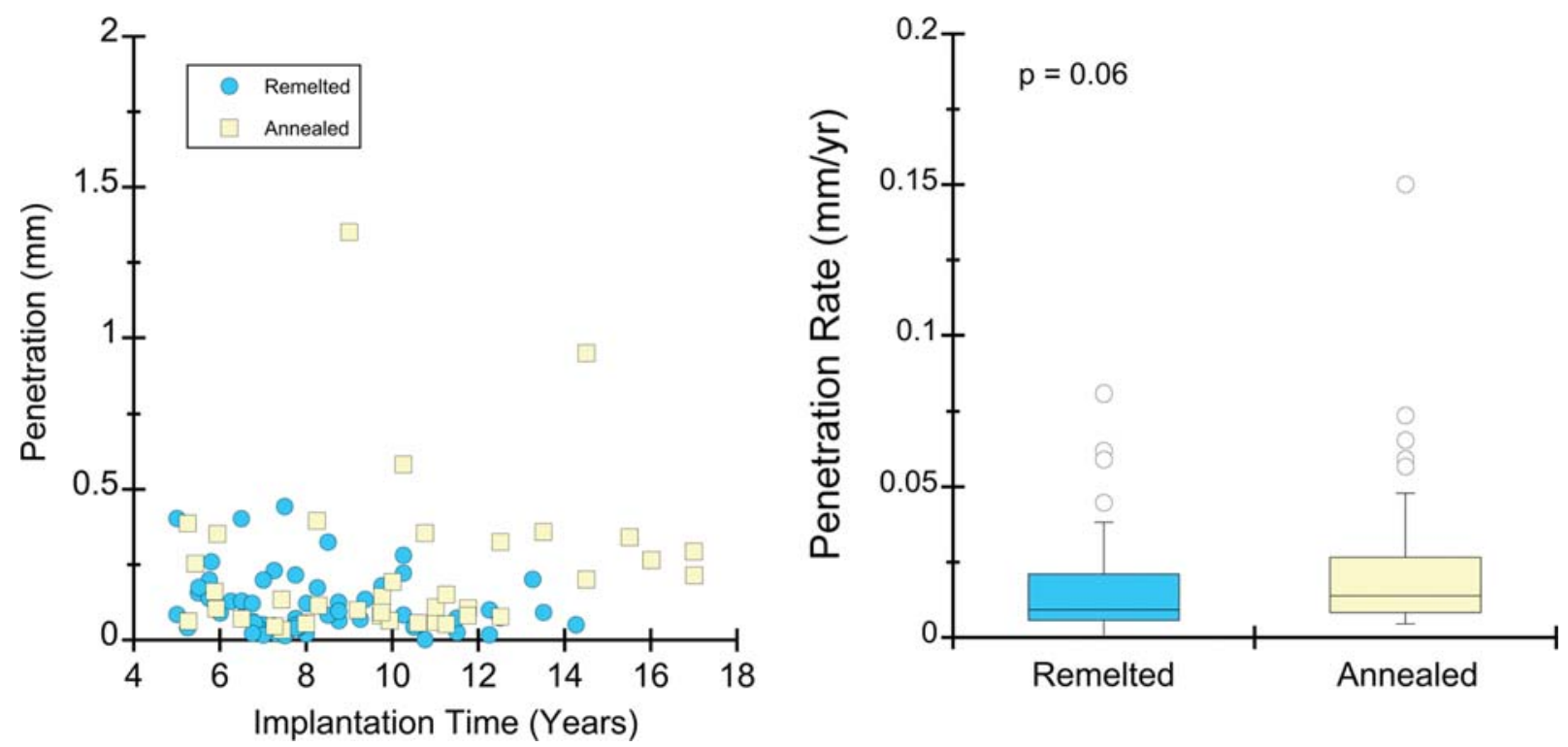

Fig. 4 Femoral head penetration (left) and femoral head penetration rates (right) were similar between the two cohorts. Thirty-one liners were not measured due to iatrogenic damage to the articular and/or backside surfaces.

correlated with femoral head size $(p=0.28$ and 0.01 for the annealed and remelted cohorts, respectively).

\section{Discussion}

Over two decades ago, HXLPEs were presented as an alternative to conventional polyethylene, providing potential increased wear resistance to decrease wear debris and subsequently reduce osteolysis. ${ }^{1}$ The purpose of this study was to determine the efficacy of these materials in reducing wear and osteolysis as well as to compare the clinical performance of the two post-irradiation thermal treatment methods. The annealed cohort had higher OIs than the remelted cohort at all ROIs: rim, backside, bearing surface, and locking mechanism. However, the femoral head penetration rate was similar between the two cohorts, and we likewise observed no differences in the reported incidence of osteolysis between annealed and remelted HXLPEs. The results of our study suggest that both annealed and remelted HXLPE formulations have similar medium to long-term clinical behavior, regardless of the fact that the annealed cohort in this study was more highly oxidized, especially at the rim.

This study had several limitations. First, the implantation time differed slightly between the two cohorts, likely due to the earlier clinical introduction of Crossfire (annealed HXLPE), which may be a confounding factor. ${ }^{1}$ However, 
when observing the oxidation of these materials, all of which had been implanted for at least 5 years, it was determined that implantation time did not correlate with the measured OIs. Additionally, all components were from revised THAs and thus may not reflect the behavior of successful components still in vivo.

The principal reasons for revision were infection, instability, and loosening, although they were found in different proportions within each cohort. Specifically, infection was the primary revision reason in $24 \%$ of the remelted cohort, whereas infection was only present in $9 \%$ of the annealed cohort. It is unclear what accounts for the increased infection observed in the remelted cohort; however, it could be the shorter implantation time, different approaches to classifying revision reasons between surgeons, or different referral patterns between the performance sites in this study. These differences may be attributed to the fact that the annealed cohort was in vivo for a longer period of time due to the earlier date of clinical availability. The predominant reasons for revision (loosening, instability, and infection) found in this study were similar to a recent retrieval study of firstand second-generation HXLPEs ${ }^{13}$ as well as a database study of THA revision reasons in the United States. ${ }^{14}$ Additionally, there were no incidences of liner fracture, as is thought to be a concern with remelted HXLPE due to diminished mechanical properties. ${ }^{15}$ This is most likely because remelted liner fractures typically occurred early, within 5 years of implantation, as a result of various factors, including being implanted vertically. ${ }^{16}$ In this study of long-term performance, these short-term failed components were excluded from our analyses.

This study demonstrated that remelted HXLPE is more oxidatively stable than annealed HXLPE, but this finding was not associated with the length of time that the liners were implanted. The nature of annealed HXLPE to have greater oxidation in vivo results from the residual free radicals, which are still detectable, even after post-irradiation thermal processing has taken place. ${ }^{1}$ Residual free radicals can lead to oxidation and chain scission when they come into contact with oxygen and will result in a decrease in the mechanical properties of the polymer. ${ }^{17}$ Regional differences were seen within both cohorts. The remelted acetabular liners were found to have low OIs (indices $<1$ ) at all ROIs, but the annealed acetabular liners exhibited low OIs at the locking mechanism, bearing surface, and backside (median OI 0.3, 0.4, and 0.3, respectively), whereas, in contrast, the rim of annealed liners was found to have higher indices (median $\mathrm{OI}=5.2$ ). These differences are presumably due to differences in the areas of contact on the liner between the femoral head and the metal shell. If oxidizing species in joint fluids is unable to reach the polyethylene surface, oxidation is reduced. ${ }^{17}$ With the exception of the rim of the annealed cohort, these indices were below the ASTM threshold OI of 1, above which oxidation is considered to substantially degrade mechanical behavior. ${ }^{13}$ Despite the rims of the annealed cohort exhibiting high OIs, we did not observe any cases of rim fracture or mechanical failure of the annealed liners. This suggests that oxidation does not appear to be a clinical concern in either cohort, at least for liners implanted for up to 17 years.

In this study, we found head penetration rates to be similar between the cohorts. This finding was consistent with a similar shorter term study comparing the two materials. ${ }^{18}$ This is likely due to the two materials has similar targeted crosslinking dosages and, thus, should provide similar resistance to wear. Oxidation of annealed HXLPE can reduce the mechanical properties of the liner and may ultimately decrease the wear resistance of the polymer. However, given the similar penetration rates of the two cohorts, it appears that the level of oxidation at the bearing surface observed in this study is not enough to degrade the long-term clinical wear behavior. The low penetration rates for the two HXLPE formulations found in this study are consistent with those found in other similar studies. ${ }^{19-24}$ Femoral head size did not affect the linear femoral head penetration rates in either cohort in this study. This is similar to a review article that found several studies where linear penetration rate is independent of femoral head size. ${ }^{25}$ However, for a given linear penetration rate, larger heads will release a larger volume of HXLPE debris into the joint space. Importantly, linear femoral head penetration rates were similar in patients with and without osteolysis. This highlights that osteolysis is a complex, multifactorial problem that is dependent on more than just linear penetration rates of the polyethylene liner.

HXLPE was developed to target the issue of polyethylene wear-induced osteolysis. Multiple studies have shown positive survivorship of HXLPE. ${ }^{3,6,7,9,14,26,27}$ However, it has yet to be determined if the incidence of osteolysis has been improved and if there is a difference in osteolysis occurrence between the two post-irradiation thermal processing methods. This study provided new information about the mid- to long-term performance (specifically the clinical reasons for revision, oxidation, and wear) of these materials as it captured a longer period of implantation as well as an increased cohort size. ${ }^{6,19-22,28-30}$ Even with the extended period of implantation, no difference in the prevalence of osteolysis was found between annealing and remelting. This result is consistent with the lack of a difference found between femoral penetration rates as volume of wear debris is an important factor in the development of osteolysis. ${ }^{31,32}$ The low incidence of osteolysis observed in this study is consistent with previous studies. ${ }^{3,21}$ Despite the low level of wear, osteolysis was still reported in a few cases, confirming that the development of osteolysis is a multifactorial problem and not solely dependent on the volume of polyethylene wear. $^{33,34}$ Of the 28 patients for which osteolysis was reported, seven observations of osteolysis were not found in the acetabular region, indicating that they may have been a result of nonpolyethylene debris, such as metal. ${ }^{33}$ Additionally, these results indicate that despite the oxidation of annealed HXLPE, it does not appear, within this cohort, to have impacted the prevalence of osteolysis. This result suggests that polyethylene wear has decreased to a point where it may no longer be impacting time to revision. In a study performed by Ollivier et $\mathrm{al}^{35}$ patients were also separated into two groups by activity score. Conventional 
UHMWPE was evaluated in Ollivier's study, and the low activity group was found to have a higher chance of survival. ${ }^{35}$ These differing results indicate that due to the increased wear resistance of HXLPE polyethylene, high activity level and the resulting increase in wear potentially may be less of a clinical concern.

In summary, this multi-institutional implant retrieval study did not find a difference in femoral head penetration rates or prevalence of osteolysis in patients between annealed and remelted HXLPE liners in total hip arthroplasty, at least not in the liners and patients analyzed in this study which were in vivo between 5 and 17 years. It remains to be seen whether HXLPE formulation has an effect on the wear performance of THA beyond 15 years or whether these formulation differences are clinically meaningful in more highly stressed total joints such as the knee.

\section{Funding}

This study was supported by the U.S. Department of Health and Human Services, National Institutes of Health, National Institute of Arthritis and Musculoskeletal and Skin Diseases (R01 AR47904).

\section{Conflict of Interest}

Dr. Klein reports grants from Zimmer Biomet and Corentec, outside the submitted work. In addition, Dr. Klein has a patent Zimmer Biomet with royalties paid, a patent Corentec with royalties paid, and a patent JayPee Brothers Medical Publishers with royalties paid. Mr. MacDonald reports grants from the National Institutes of Health, during the conduct of the study, grants from Stryker Orthopedics, personal fees from CeramTec, outside the submitted work. Dr. Malkani reports grants and personal fees from Stryker, outside the submitted work. Dr. Kraay has nothing to disclose. Dr. Mont reports personal fees from Cymedica, grants and personal fees from DJ Orthopaedics, personal fees from Flexion Therapeutics, grants and personal fees from Johnson \& Johnson, personal fees from Medicus Works LLC, personal fees from MicroPort, grants and personal fees from Ongoing Care Solutions, grants and personal fees from Orthosensor, personal fees from Pacira, personal fees from PeerWell, personal fees from Performance Dynamics, personal fees from Pfizer, personal fees from Skye Biologics, grants and personal fees from Stryker, grants and personal fees from Tissue Gene, outside the submitted work. Dr. Kurtz reports grants from the National Institutes of Health, during the conduct of the study; grants from Celanese, grants from CeramTec, grants from Ferring Pharmaceuticals, personal fees from Exponent, grants from Invibio, grants from Simplify Medical, grants from Lima Corporate, grants from Stryker, grants from Wright Medical Technology, grants from Zimmer Biomet, outside the submitted work. Dr. Lee reports personal fees from Stryker, personal fees from Corin, personal fees from Heron Therapeutics, grants from Smith \& Nephew, grants from KCI Acelity, grants from Ferring, outside the submitted work.

\section{Acknowledgments}

This study was made possible by the National Institutes of Health (R01 AR47904). We would also like to thank Genymphas Higgs for assistance with the data analysis in this study. We would also like to thank Drs. Harold Cates and Brian Hamlin for providing retrieved devices and operative reports used in this study. We would also like to acknowledge the Wilbert J. Austen Professor of Engineering Chair (CMR).

\section{References}

1 Kurtz SM. UHMWPE Biomaterials Handbook: Ultra High Molecular Weight Polyethylene in Total Joint Replacement and Medical Devices. London: Academic Press; 2009

2 Kurtz SM, Hozack W, Turner J, et al. Mechanical properties of retrieved highly cross-linked crossfire liners after short-term implantation. J Arthroplasty 2005;20(07):840-849

3 Kurtz SM, Gawel HA, Patel JD. History and systematic review of wear and osteolysis outcomes for first-generation highly crosslinked polyethylene. Clin Orthop Relat Res 2011;469(08): 2262-2277

4 Wannomae KK, Bhattacharyya S, Freiberg A, Estok D, Harris WH, Muratoglu O. In vivo oxidation of retrieved cross-linked ultrahigh-molecular-weight polyethylene acetabular components with residual free radicals. J Arthroplasty 2006;21(07): 1005-1011

5 Atwood SA, Van Citters DW, Patten EW, Furmanski J, Ries MD, Pruitt LA. Tradeoffs amongst fatigue, wear, and oxidation resistance of cross-linked ultra-high molecular weight polyethylene. J Mech Behav Biomed Mater 2011;4(07):1033-1045

6 Capello WN, D’Antonio JA, Ramakrishnan R, Naughton M. Continued improved wear with an annealed highly cross-linked polyethylene. Clin Orthop Relat Res 2011;469(03):825-830

7 Thomas GE, Simpson DJ, Mehmood S, et al. The seven-year wear of highly cross-linked polyethylene in total hip arthroplasty: a double-blind, randomized controlled trial using radiostereometric analysis. J Bone Joint Surg Am 2011;93(08):716-722

8 Engh CA Jr, Hopper RH Jr, Huynh C, Ho H, Sritulanondha S, Engh CA Sr. A prospective, randomized study of cross-linked and noncross-linked polyethylene for total hip arthroplasty at 10 -year follow-up. J Arthroplasty 2012;27(08):2-7.e1

9 Kurtz SM, Austin MS, Azzam K, et al. Mechanical properties, oxidation, and clinical performance of retrieved highly crosslinked Crossfire liners after intermediate-term implantation. J Arthroplasty 2010;25(04):614-23.e1, 2

10 James SP, Blazka S, Merrill EW, et al. Challenge to the concept that UHMWPE acetabular components oxidize in vivo. Biomaterials 1993;14(09):643-647

11 International A. Standard Guide for Evaluating the Extent of Oxidation in Polyethylene Fabricated Forms Intended for Surgical Implants. Vol ASTM Standard F2101-13. West Conshohocken, PA: ASTM; 2013

12 Gómez-Barrena E, Li S, Furman BS, Masri BA, Wright TM, Salvati EA. Role of polyethylene oxidation and consolidation defects in cup performance. Clin Orthop Relat Res 1998;(352):105-117

13 Kurtz SM, MacDonald DW, Mont MA, Parvizi J, Malkani AL, Hozack W. Retrieval analysis of sequentially annealed highly crosslinked polyethylene used in total hip arthroplasty. Clin Orthop Relat Res 2015;473(03):962-971

14 Bozic KJ, Kurtz SM, Lau E, Ong K, Vail TP, Berry DJ. The epidemiology of revision total hip arthroplasty in the United States. J Bone Joint Surg Am 2009;91(01):128-133

15 Tower SS, Currier JH, Currier BH, Lyford KA, Van Citters DW, Mayor MB. Rim cracking of the cross-linked longevity polyethylene 
acetabular liner after total hip arthroplasty. J Bone Joint Surg Am 2007;89(10):2212-2217

16 Halley D, Glassman A, Crowninshield RD. Recurrent dislocation after revision total hip replacement with a large prosthetic femoral head. A case report. J Bone Joint Surg Am 2004;86(04): 827-830

17 Currier BH, Currier JH, Collier JP, Mayor MB. Effect of fabrication method and resin type on performance of tibial bearings. J Biomed Mater Res 2000;53(02):143-151

18 Takada R, Jinno T, Koga D, Hirao M, Muneta T, Okawa A. Comparison of wear rate and osteolysis between annealed and remelted highly cross-linked polyethylene in total hip arthroplasty. A case control study at 7 to 10 years follow-up. Orthop Traumatol Surg Res 2016;102(06):717-721

19 McCalden RW, MacDonald SJ, Rorabeck CH, Bourne RB, Chess DG, Charron KD. Wear rate of highly cross-linked polyethylene in total hip arthroplasty. A randomized controlled trial. J Bone Joint Surg Am 2009;91(04):773-782

20 Haw JG, Battenberg AK, Huang DT, Schmalzried TP. Wear rates of larger-diameter cross-linked polyethylene at 5 to 13 years: does liner thickness or component position matter? J Arthroplasty 2017;32(04):1381-1386

21 Bitsch RG, Loidolt T, Heisel C, Ball S, Schmalzried TP. Reduction of osteolysis with use of Marathon cross-linked polyethylene. A concise follow-up, at a minimum of five years, of a previous report. J Bone Joint Surg Am 2008;90(07):1487-1491

22 Babovic N, Trousdale RTMD. Total hip arthroplasty using highly cross-linked polyethylene in patients younger than 50 years with minimum 10-year follow-up. J Arthroplasty 2013;28(05):815-817

23 Shia DS, Clohisy JC, Schinsky MF, Martell JM, Maloney WJ. THA with highly cross-linked polyethylene in patients 50 years or younger. Clin Orthop Relat Res 2009;467(08):2059-2065

24 García-Rey E, García-Cimbrelo E, Cruz-Pardos A. New polyethylenes in total hip replacement: a ten- to 12-year follow-up study. Bone Joint J 2013;95-B(03):326-332

25 Cross MB, Nam D, Mayman DJ. Ideal femoral head size in total hip arthroplasty balances stability and volumetric wear. HSS J 2012;8 (03):270-274
26 Garvin KL, White TC, Dusad A, Hartman CW, Martell J. Low wear rates seen in THAs with highly crosslinked polyethylene at 9 to 14 years in patients younger than age 50 years. Clin Orthop Relat Res 2015;473(12):3829-3835

27 Olyslaegers C, Defoort K, Simon JP, Vandenberghe L. Wear in conventional and highly cross-linked polyethylene cups: a 5-year follow-up study. J Arthroplasty 2008;23(04):489-494

28 Dorr LD, Wan Z, Shahrdar C, Sirianni L, Boutary M, Yun A. Clinical performance of a Durasul highly cross-linked polyethylene acetabular liner for total hip arthroplasty at five years. J Bone Joint Surg Am 2005;87(08):1816-1821

29 Rajadhyaksha AD, Brotea C, Cheung Y, Kuhn C, Ramakrishnan R, Zelicof SBMDP. Five-year comparative study of highly cross-linked (crossfire) and traditional polyethylene. J Arthroplasty 2009;24 (02):161-167

30 Choi WK, Cho MR, Lee JH. Results of primary total hip arthroplasty with $36-\mathrm{mm}$ femoral heads on highly cross-linked polyethyleneminimum seven-years follow-up. Hip Pelvis 2014;26(04): 220-226

31 Glyn-Jones S, Thomas GER, Garfjeld-Roberts P, et al. The John Charnley Award: highly crosslinked polyethylene in total hip arthroplasty decreases long-term wear: a double-blind randomized trial. Clin Orthop Relat Res 2015;473(02):432-438

32 Gomez-Barrena E, Puertolas JA, Munuera L, Konttinen YT. Update on UHMWPE research: from the bench to the bedside. Acta Orthop 2008;79(06):832-840

33 Huber M, Reinisch G, Zenz P, Zweymüller K, Lintner F. Postmortem study of femoral osteolysis associated with metal-on-metal articulation in total hip replacement: an analysis of nine cases. J Bone Joint Surg Am 2010;92(08):1720-1731

34 Huddleston HD. Femoral lysis after cemented hip arthroplasty. J Arthroplasty 1988;3(04):285-297

35 Ollivier M, Frey S, Parratte S, Flecher X, Argenson JN. Does impact sport activity influence total hip arthroplasty durability? Clin Orthop Relat Res 2012;470(11):3060-3066 